\title{
Respiratory syncytial virus infection of airway epithelial cells, in vivo and in vitro, supports pulmonary antibody responses by inducing expression of the $B$ cell differentiation factor BAFF
}

\author{
P S McNamara, ${ }^{1}$ A M Fonceca, ${ }_{1}^{1}$ D Howarth, ${ }^{2}$ J B Correia, ${ }^{3}$ J R Slupsky, ${ }_{1}^{4}$ R E Trinick, ${ }^{1}$ \\ W Al Turaiki, ${ }^{1}$ R L Smyth, ${ }^{1}$ B F Flanagan ${ }^{1}$
}

'Department of Women's and Children's Health, Institute of Translational Medicine, University of Liverpool, Alder Hey Children's NHS Foundation Trust Hospital, Liverpool, UK ${ }^{2}$ Department of Clinical Infection, Microbiology and Immunology, Institute of Infection and Global Health, University of Liverpool,

Liverpool, UK

${ }^{3}$ Instituto de Medicina Integral Professor Fernando Figueira (IMIP), Recife, Brazil

${ }^{4}$ Molecular and Clinical Cancer Medicine, Institute of Translational Medicine, University of Liverpool, Liverpool, UK

\section{Correspondence to}

Dr B F Flanagan,

Department of Women's and Children's Health, Institute of Translational Medicine, University of Liverpool, Alder Hey Children's NHS Foundation Trust Hospital, Eaton Road, Liverpool, L12 2AP, UK; fla1@liv.ac.uk

PSM and AMF contributed equally

Received 15 June 2012 Revised 31 July 2012 Accepted 20 August 2012 Published Online First 21 September 2012

\section{SLinked}

- http://dx.doi.org/10.1136/ thoraxjnl-2012-202182 - http://dx.doi.org/10.1136/ thoraxjnl-2012-202291

To cite: McNamara PS Fonceca AM, Howarth $D_{\text {, }}$ et al. Thorax 2013, 68, 76-81.

\section{ABSTRACT}

Background The mechanisms regulating antibody expression within the human lung during airway infection are largely unknown. In this study, our objectives were to determine if infection with respiratory syncytial virus (RSV) upregulates expression of the B cell differentiation factors $A$ proliferation inducing ligand (APRIL) and B cell activating factor of the TNF family (BAFF), if this is a common feature of viral airway infection, and how this is regulated in human airway epithelial cells.

Methods We measured BAFF and APRIL protein expression in bronchoalveolar lavage (BAL) fluid from infants with severe RSV disease, and healthy control children, and in nasopharyngeal aspirates from preschool children with other single respiratory viral infections. We also measured mRNA expression in bronchial brushings from RSV-infected infants, and in RSV-infected paediatric primary airway epithelial cell cultures (pAEC). Beas-2B cell cultures were used to examine mechanisms regulating BAFF expression.

Results BAFF protein and mRNA were elevated (in marked contrast with APRIL) in BAL and bronchial brushings, respectively, from RSV-infected infants. BAFF protein was also found in upper airway secretions from children with human metapneumovirus, H1N1, bocavirus, rhinovirus, RSV and Mycoplasma pneumoniae infection. BAFF mRNA and protein were expressed following in vitro RSV infection of both pAEC and Beas2B cultures, with mRNA expression peaking 12-h postinfection. BAFF induction was blocked by addition of a neutralising anti-interferon- $\beta$ antibody or palivizumab. Conclusions BAFF, produced through an interferon- $\beta$ dependant process, is a consistent feature of airway infection, and suggests a role for the airway epithelia in supporting protective antibody and B cell responses in the lung.

\section{INTRODUCTION}

Respiratory syncytial virus (RSV) is a major worldwide cause of respiratory tract infections in young children, with severe airway inflammation and mucus hypersecretion leading to death in approximately $2 \%$ of admitted cases. ${ }^{1}$ RSV preferentially infects and replicates in the airway epithelium. As in other airway viral infections, innate immune mediators expressed by normal stromal cells,

\section{Key messages}

What is the key question?

- What role does the human airway epithelium have in supporting the protective antibody response to respiratory viral infections, such as respiratory syncytial virus (RSV) in young children?

\section{What is the bottom line?}

- During RSV infection, and probably other respiratory viral infections, increased expression of the $B$ cell differentiation factor $B$ cell activating factor of the TNF family (BAFF) within primary airway epithelial cells occurs in an interferon $\beta$-dependant manner, demonstrating the potential for the airway epithelium to support protective B lymphocyte responses.

\section{Why read on?}

- Despite the known importance of the antibody in protecting the human airway, few studies have addressed how B cell responses are stimulated, or supported in the lung, this study emphasises the importance of the airway epithelia in influencing immune and inflammatory responses in the surrounding lung tissues, by demonstrating that it responds to viral infection by producing the key $B$ cell differentiation factor BAFF.

including infected airway epithelia, influence both early inflammatory responses and the subsequent development of an adaptive immune response. ${ }^{2-4}$ Neutrophils account for the majority, 80\%-90\%, of cells recruited into the airways, lymphocytes $6 \%-10 \%$, with B cells reported to be the major lymphocyte population present within the lung. ${ }^{56}$

Mediators that promote production and expression of protective antibody during infections in the human lung are very poorly understood. It is important to have a better understanding of antibody induction in the airways, not only for protecting against respiratory infection, but to improve strategies for airway vaccine design. A previous 
study of human RSV infection ${ }^{7}$ identified two molecules in the lung, B cell activating factor of the TNF family, TNFSF13B (BAFF), and A proliferation including ligand, TNFSF13 (APRIL), with the potential to influence B cell maturation, proliferation and antibody class switch recombination to Immunoglobulin A $(\operatorname{IgA}){ }^{8-12}$

RSV-specific neutralising IgA can be found in the lung at 3-5 days postinfection. ${ }^{13}$ The presence of maternal $\operatorname{IgA}$ in infants, and high levels of nasal RSV-specific IgA in adults, has been shown to correlate with protection from infection. ${ }^{14}{ }^{15} \mathrm{In}$ an animal model of RSV infection, an IgA monoclonal antibody applied nasally, protected against upper and lower respiratory tract infection. ${ }^{16}$ However, high antibody levels do not always correlate with protection from disease. Postinfection antibody levels decline progressively and have been suggested to predispose individuals to subsequent reinfection. ${ }^{16}$

Here, we sought to establish whether BAFF or APRIL expression characterise viral infection of the airways in children. Specifically, we investigated whether elevated expression of these mediators was seen in the lower airways of infants with severe RSV bronchiolitis, and in the upper airways of preschool children with other viral and atypical bacterial respiratory infections. Furthermore, we investigated if viral infection of cultured primary airway epithelial cells (AEC) in vitro is sufficient to induce expression, and whether induction is mediated directly by viral infection or dependant on interferon $\beta$ expression by infected cells.

\section{METHODS}

\section{Patient samples}

Non-bronchoscopic bronchial brushings and bronchoalveolar lavage fluid (BALF) were collected from healthy non-atopic children undergoing elective surgery for non-respiratory conditions, as described previously. ${ }^{6} 17$ The absence of respiratory symptoms was confirmed by negative responses on the International Study of Asthma and Allergies in Childhood and American Thoracic Society respiratory questionnaires. Samples were also collected from clinically stable infants ventilated with RSV bronchiolitis, born at term, with no underlying medical problems. RSV status was ascertained by enzyme-linked fluorescence (ELFA, Biomerieux, Marcy l'Etoile, France) of nasopharyngeal aspirate. We collected samples within $24 \mathrm{~h}$ of admission, just prior to routine bronchial toilet, and sample collection was well tolerated by all children. Purified AEC from bronchial brushings were stored in rapid lysis buffer (Qiagen) at $-70^{\circ} \mathrm{C}$ for gene expression analysis, and the remainder used for cell culture. BALF was aliquoted and stored at $-70^{\circ} \mathrm{C}$ until needed for analysis.

Nasopharyngeal aspirates (NPA) were collected from preschool children with acute respiratory infection at the Instituto de Medicina Integral Prof Fernando Figueira in Recife, Pernambuco, North-East Brazil, as described previously. ${ }^{18}$ Real-time PCR analysis for 18 viral and atypical bacterial respiratory pathogens (RSV, Rhinovirus (RhV), human metapneumovirus (hMPV), influenza viruses (A (including H1N1) and B), parainfluenza viruses (1-4), coronaviruses (OC43, NL63, 229E, HKU1), adenovirus, bocavirus, Mycoplasma pneumoniae and Chlamydia pneumoniae) was performed on each sample collected using standard published diagnostic protocols as described previously. ${ }^{18}$ Only samples in which one of the following pathogens was detected were included in the study; RSV, RhV, H1N1, bocavirus, hMPV and M pneumoniae.

Ethics committees in both Liverpool and Recife approved the study, and all samples were collected following written informed consent from the parents.

\section{RSV propagation}

RSV A2 was cultivated, harvested and titrated using HEp-2 monolayers as described previously. ${ }^{2}$

\section{AEC culture}

Primary AECs from healthy children were cultured until the second passage as described previously. ${ }^{19}$ Primary cultures with varying titres $(0.0025,0.025,0.25$ and 2.5 multiplicities of infection (MOI)) of RSV and harvested following incubations of 3, 12 and $48 \mathrm{~h}$. Filtered RSV viral stock $(30 \mathrm{kDa})$ and noninfected cultures and palivizumab-treated cultures were used as controls. Cell pellets were collected for RNA preparation and supernatants aliquoted and stored at $-70^{\circ} \mathrm{C}$.

BAFF induction was also assessed in Beas $2 \mathrm{~B}$ cell cultures infected with RSV with and without palivizumab (1.6 ug/ml) (SYNAGIS, Abbott Laboratories, Maidenhead, UK), and treated with IFN- $\beta$ (10 and $100 \mathrm{U} / \mathrm{ml}$ ) (PBL Interferon Source, Piscataway, NJ, USA). Anti-IFN- $\beta$ neutralising antibody $(500 \mathrm{U} / \mathrm{ml})$ (PBL Interferon Source) was used to assess response specificity.

\section{APRIL, BAFF and IL-8 expression analysis}

Total RNA was extracted from patient samples and culture cell pellets using the RNeasy kit (Qiagen, Manchester, UK), and $2 \mu \mathrm{g}$ of total RNA used as a template for cDNA synthesis using random primers and M-MLV reverse transcriptase (Promega, Southampton, UK). BAFF, APRIL and IL-8 mRNA expression were determined by Taqman real-time PCR normalised to expression of the housekeeping gene L32 as described by the manufacturer (Applied Biosystems, Warrington, UK). Secreted cytokines levels were measured using commercially available ELISA kits (R\&D Systems, Oxford, UK) according to the manufacturer's instructions.

\section{Statistical analysis}

Data from RSV-infected and healthy patient samples were expressed as medians (IQRs) with statistical differences between groups assessed using the Mann-Whitney U test.

All in vitro experiments were completed in duplicate with a minimum of three biological replicates. In each case, duplicate results were averaged to give an experimental value that was in turn averaged across replicate experiments. The final mean replicate value with SEM are presented unless otherwise stated. Experiments involving cell cultures were tested for differences using the Student $t$ test with significance taken at $\mathrm{p}<0.05$. Significance is indicated by an asterisk in all figures unless stated otherwise.

\section{RESULTS}

\section{Clinical characteristics}

Non-bronchoscopic bronchial brushings and BALF were collected from 20 healthy non-atopic children $(9$ male; median (range) age, 8 (2-15) years). Samples were also collected from 13 clinically stable infants (5 male; age, 4 (2-37) weeks) ventilated with RSV disease. These infants were hospitalised with clinical features of bronchiolitis (apnoea, 4/13) having had a mean (SD) duration of illness of 5.0 (2.7) days, and were ventilated for 90 (28) h. Parents of seven infants with bronchiolitis gave consent for both bronchial brushing and bronchoalveolar lavage (BAL), while parents of six infants gave consent for only BAL to be performed. The mean (SEM) bronchial brushing cell yield from healthy and RSV children was $3.4(1.2) \times 10^{6}$ and $2.6(1.1) \times 10^{6}$ cells, respectively. Mean (SEM) BAL cell yield 
Figure 1 B cell activating factor of the TNF family (BAFF) production increases in the human airway during respiratory syncytial virus (RSV) infection. (A) BAFF and A proliferation inducing ligand (APRIL) protein concentration in bronchoalveolar lavage fluid from infants with severe RSV bronchiolitis $(n=12)$ and healthy control subjects $(n=7)\left({ }^{*} p<0.001\right)$ and (B) BAFF and APRIL mRNA expression in freshly isolated airway epithelial cells from infants with severe RSV bronchiolitis $(n=6)$ and healthy control subjects $(n=9)$. Values shown as $\%$ expression in comparison with $\mathrm{L} 32$
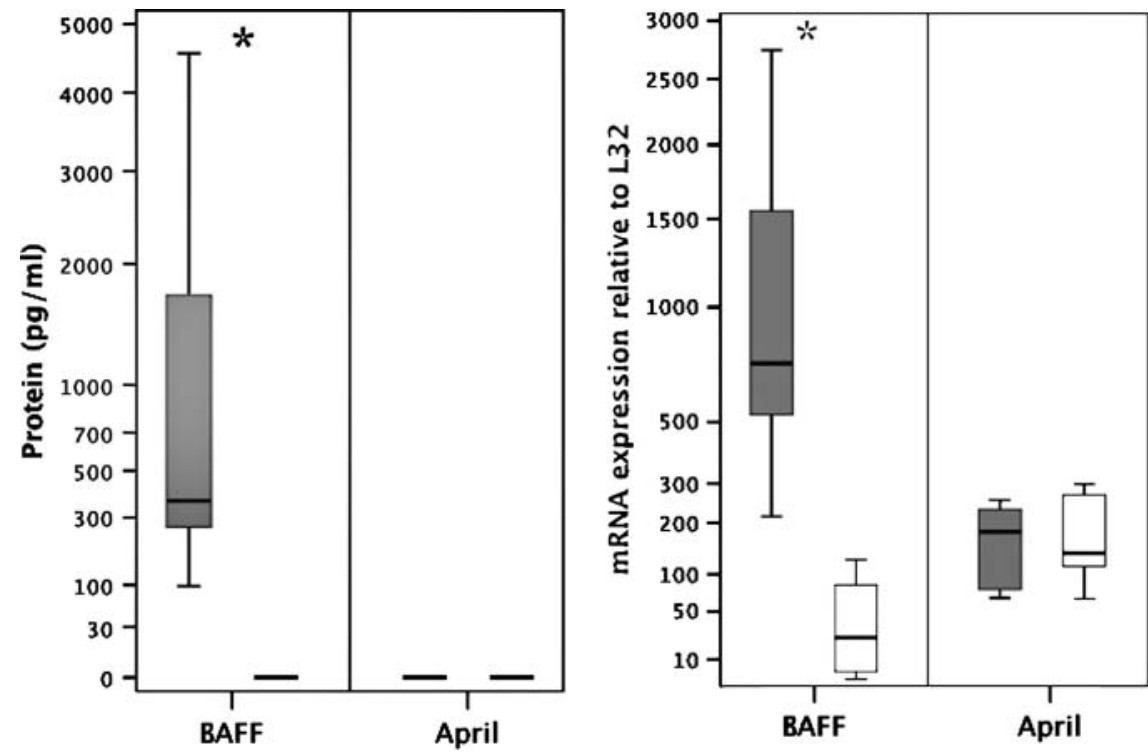

from healthy and RSV children was $2.1(0.2) \times 10^{6}$ cells and 5.3 $(3.0) \times 10^{6}$ cells, respectively.

NPA were collected from 78 children $<5$ years old with different clinical manifestations of Acute Respiratory Infection (ARI) (bronchiolitis $(n=44)$, pneumonia $(n=25)$, viral-induced wheeze $(\mathrm{n}=7)$, Upper Respiratory Tract Infection (URTI) $(\mathrm{n}=2))$. Single infections of RSV $(\mathrm{n}=38), \operatorname{RhV}(\mathrm{n}=8), \mathrm{H} 1 \mathrm{~N} 1$ $(\mathrm{n}=5)$, bocavirus $(\mathrm{n}=9)$, hMPV $(\mathrm{n}=9)$ and $M$ pneumoniae $(\mathrm{n}=9)$ were detected by RT-PCR.

BAFF, but not APRIL protein and mRNA expression is increased in the airways during severe RSV bronchiolitis, compared with health infants

To determine if BAFF or APRIL protein expression in vivo were upregulated in the lower airway during ongoing human RSV disease, we measured both BAFF and APRIL concentrations in BAL fluid from infants with severe RSV bronchiolitis $(n=12)$, and compared these with BAL from the lungs of healthy children $(n=7)$. BAFF protein was significantly upregulated $(\mathrm{p}<0.001)$ with a median of 364 (range 7-4556) pg/ml, in comparison with control concentrations median $0(0-13) \mathrm{pg} / \mathrm{ml}$. APRIL protein concentrations, in contrast, were below the minimum sensitivity of the assay in all samples $(400 \mathrm{pg})$ (figure 1A). To investigate in vivo expression of BAFF, and to determine if AECs in vivo were a possible source of elevated BAFF protein, we compared BAFF and APRIL mRNA expression in freshly isolated bronchial brushings from both RSV-infected and control group infants (figure 1B). Expression of BAFF mRNA was significantly elevated, by a factor of around 31-fold in comparison with controls $(\mathrm{p}=0.001)$. Median BAFF mRNA expression in RSV-infected AECs from babies with bronchiolitis was 740 (range 216-2742), and for healthy controls, 24 (2-126). APRIL mRNA was expressed in both groups, but levels were not significantly elevated in the RSV group compared with the control group. Median APRIL mRNA expression in RSV-infected infants was 184 (range 66-257), and in healthy controls, 138 (range 65-683): $\mathrm{p}=0.814$ (figure 1B).

\section{BAFF protein is expressed in a wide range of respiratory infections}

We next determined if elevated BAFF expression was a common characteristic of the immune response to respiratory tract infection or specific to RSV disease. We therefore looked at BAFF expression in NPA samples collected from children with hMPV $(\mathrm{n}=9)$, H1N1 flu $(\mathrm{n}=5)$, bocavirus $(\mathrm{n}=9)$, rhinovirus $(\mathrm{n}=8)$ or M pneumoniae $(\mathrm{n}=9)$ infection, as well as children with RSV infection $(n=38)$. BAFF protein was found in NPA samples from each infection (figure 2). In the RSV patient group, BAFF levels were no different in those children who did and did not need oxygen therapy (data not shown). A minimum of five samples from patients with each infection were tested for APRIL protein expression, however, this protein was not detected in any patient group.

In vitro RSV infection of primary human AECs results in increased BAFF mRNA and protein

RSV in vivo infects and replicates within airway epithelia. ${ }^{2}$ In order to determine if RSV infection is sufficient to induce expression of BAFF or APRIL, primary AECs prepared from healthy donors were challenged with RSVA2 strain at increasing

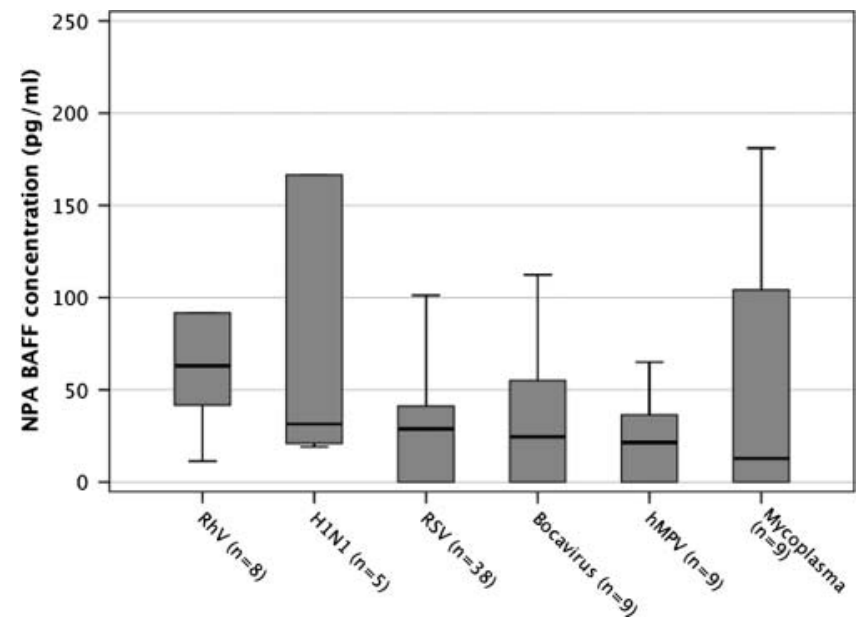

Figure 2 B cell activating factor of the TNF family (BAFF) expression is a common feature of respiratory infection. BAFF protein expression in nasopharyngeal aspirates from preschool children infected with rhinovirus $(n=8), H 1 N 1$ influenza $(n=5)$, respiratory syncytial virus $(n=38)$, bocavirus $(n=9)$, human metapneumovirus $(n=9)$ and mycoplasma $(n=9)$. 
MOI from 0.0025 to 2.5 in culture for 3,12 or $48 \mathrm{~h}$ and compared with non-infected or cells mock-infected with viral stocks filtered to remove virus. BAFF, APRIL and IL-8 mRNA levels were first measured in comparison with the housekeeping gene, L32, by Taqman real-time PCR. IL-8 mRNA expression increased progressively with increasing RSV MOI (figure 3A). BAFF mRNA levels were significantly elevated at $12 \mathrm{~h}$ post-RSV infection when compared with non-infected cells or cells treated with filter-sterilised virus preparation $(p=0.001)$ (figure $3 \mathrm{~A}$ ). Cells prepared from four separate donors each responded by producing BAFF; average fold induction was 4319 with a range from 37 to 17 115). Peak BAFF mRNA expression was induced at an MOI of 0.25 , which was also optimal for RSV RNA expression (data not shown). By contrast with BAFF mRNA, although APRIL mRNA was again constitutively expressed, we did not observe significantly increased APRIL mRNA expression at 3,12, 48 (figure $3 \mathrm{~A}$ ) or $24 \mathrm{~h}$ (data not shown) post-RSV infection by these AECs.

Figure 3 B cell activating factor of the TNF family (BAFF) but not $A$ proliferation inducing ligand (APRIL) mRNA expression and protein production, is increased following respiratory syncytial virus (RSV) infection of cultured primary airway epithelial cells (AECs). (A) BAFF, APRIL and IL8 mRNA expression were analysed by Taqman real-time PCR at 3, 12 and $48 \mathrm{~h}$ following RSV infection (multiplicities of infection (MOI) $0.0025-2.5)$. All values were normalised against expression of the L32 house-keeping gene and expressed as fold induction relative to gene expression in the filter control.

(B) BAFF protein concentrations in culture AEC supernatants, $48 \mathrm{~h}$ after RSV infection (MOI 0.025-2.5).

A
As shown in figure $3 \mathrm{~B}$, supernatants of virus-infected cultures contained up to $200 \mathrm{pg} / \mathrm{ml}$ of soluble BAFF protein $48 \mathrm{~h}$ postinfection. Soluble BAFF protein was not detected in control cultures, either non-stimulated, or stimulated with filter-sterilised viral stock. APRIL protein was not detected in either infected or non-infected primary AEC culture supernatants.

\section{BAFF expression following RSV infection is dependent on interferon $\boldsymbol{\beta}$}

In order to determine if BAFF expression was directly induced by RSV infection, or if it was indirectly dependant on interferon $\beta$ production by infected cells, we compared BAFF expression by Beas $2 \mathrm{~B}$ cells in the presence or absence of an anti-interferon $\beta$-neutralising polyclonal antisera. In these experiments, mRNA expression was measured $12 \mathrm{~h}$ postinfection (figure 4). BAFF mRNA expression was induced by RSV infection and by addition of interferon $\beta$. Treatment with neutralising anti-interferon $\beta$ antibody inhibited both RSV and interferon $\beta$-induced BAFF

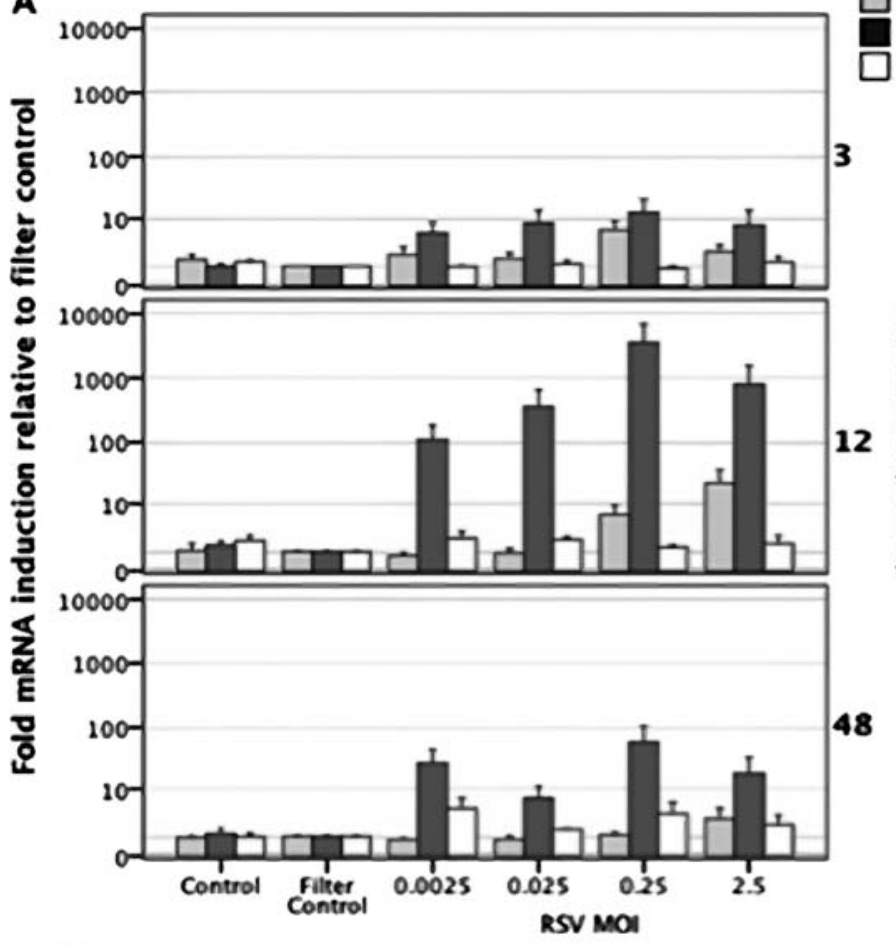

B

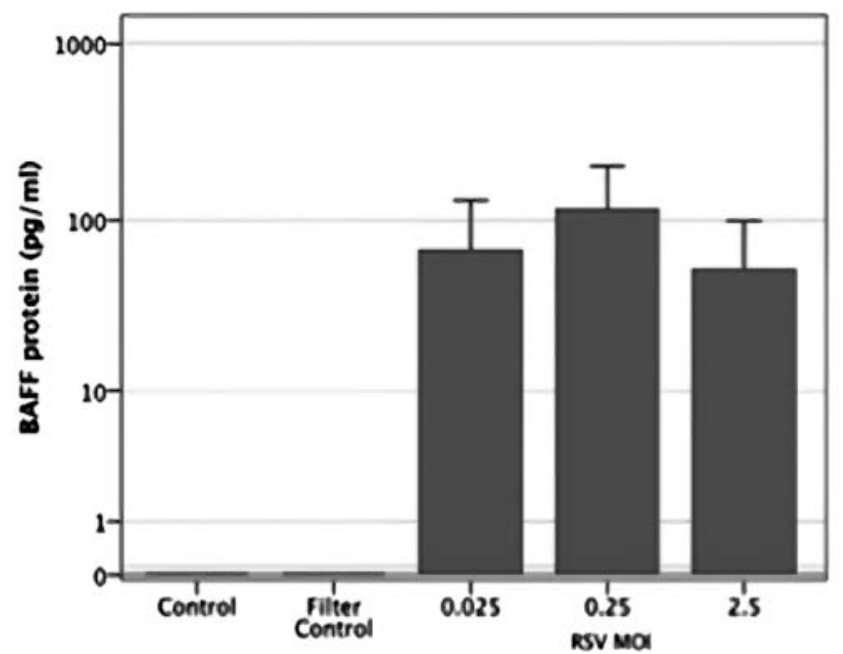

IL-8 Expression BAFF Expression April Expression

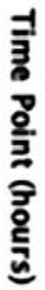




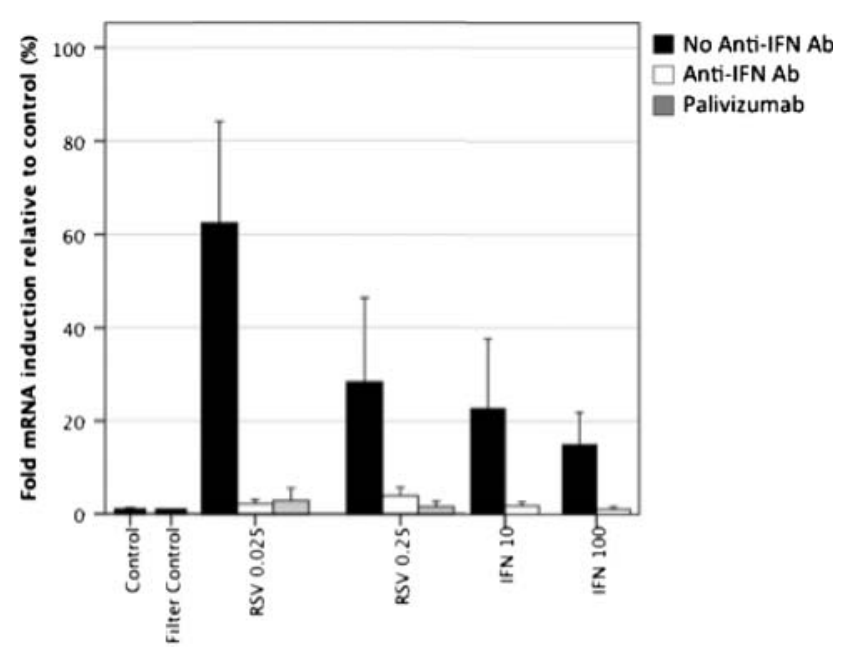

Figure 4 B cell activating factor of the TNF family (BAFF) expression following respiratory syncytial virus (RSV) infection is dependent on interferon beta. Beas-2B cells were either infected with RSV at multiplicities of infection of 0.025 and 0.25 or stimulated with 10 or 100 units of interferon $\beta$. Matched samples contained the same stimuli with addition of either a neutralising polyclonal antihuman interferon $\beta$ antibody or the neutralising anti-RSV recombinant monoclonal antibody, palivizumab. BAFF mRNA expression was measured at $12 \mathrm{~h}$ after stimulation, values are shown as fold expression in comparison with L32.

expression, with observed values closely resembling those of the control cultures. Similarly, preincubation with a monoclonal antibody to the RSV fusion protein (palivizumab) abolished RSV-induced BAFF mRNA expression, further confirming viral specific induction of expression.

\section{DISCUSSION}

Although it is well known that innate signals from nonlymphoid cells influence the production, magnitude and type of T cell response in the lung, ${ }^{4}$ few studies to date have focused on the factors that regulate $\mathrm{B}$ cell responses. Here, we have shown for the first time, BAFF protein expression in BAL fluid and BAFF mRNA in AEC from bronchial brushings in the lower airways of infants with severe RSV bronchiolitis. We have also found BAFF protein in the upper airways of children with other single viral and atypical bacterial infections, suggesting that its production occurs throughout the respiratory tract and is not RSV-specific but a common feature of the airway response to infection. In vitro, RSV infection of cultured primary AECs resulted in greatly increased BAFF production relative to IL-8 and APRIL. Lastly, we have shown that BAFF expression is interferon-dependent by inducing BAFF expression with interferon $\beta$, and by blocking RSV-induced BAFF production with a neutralising anti-interferon $\beta$ antibody. These results demonstrate the importance of the epithelium, but do not exclude the possibility that other cells within the lung, such as inflammatory cells, may also produce B cell differentiation factors.

Although expression of BAFF has been reported in autopsy samples from RSV-infected infants, ${ }^{7}$ this is the first study to show direct upregulation by isolated AECs in response to viral infection. To model as closely as possible RSV infection within the human airway, ${ }^{2}$ we used primary AECs from the lungs of children. In these cultured cells, BAFF gene expression was consistently upregulated in response to RSV, peaking much higher than either IL-8 or APRIL expression, some 3500 times greater than filter control. We argued that this sizeable response could be due to AEC indirect activation by inflammatory signals, such as Type 1 interferon, ${ }^{20}{ }^{21}$ and so examined this possibility in an immortalised epithelial cell-line. In BEAS-2Bs, we were able to stimulate BAFF expression using interferon $\beta$, and to block RSV-induced BAFF expression using an anti-interferon antibody, indicating that early BAFF AEC expression following RSV infection, at the very least, is dependent on interferon $\beta$ production. As interferon is a common component of the immune response to many viral infections, we propose that the BAFF expression associated with the other infections reported here might also be interferon-dependant. Also notable in these experiments is the greater induction of BAFF expression, around 100-fold higher in primary airway cells in comparison with BEAS-2Bs, further confirming the enhanced cytokine production of primary cells. ${ }^{2}$

Many pathogenic viruses have been reported to inhibit the host cell Type 1 interferon response to infection. For RSV, it has previously been demonstrated that NS1 and NS2 proteins once expressed within an infected cell, act to inhibit, but not abolish, interferon production during infection, 22 and can reduce both innate and $\mathrm{T}$ cell-mediated immunity. ${ }^{24} 25$ In vitro, we were able to induce BAFF with interferon $\beta$ at concentrations as low as 10 units $/ \mathrm{ml}$. We also observed that BAFF mRNA expression and protein production in primary RSV-infected AECs lessened at higher RSV MOIs. Although this could have been due to the direct cytotoxic effect of the virus on the AECs, it is also possible that interferon inhibition by RSV NS proteins was greater at higher MOIs, thus reducing AEC BAFF expression. In vivo, this overall effect would serve to reduce or delay the B cell response to RSV infection, potentially a mechanism shared with other viruses that inhibit the host interferon response.

By contrast with BAFF, neither protein or mRNA expression of the related factor APRIL was shown to be upregulated in samples from infected infants, despite APRIL mRNA being constitutively expressed at levels five times higher than BAFF in epithelium from healthy control children. These results may be explained first by the relatively low sensitivity of the APRIL ELISA which, similar to other available assays, was around 400 pg. Alternatively, APRIL is known to be expressed at the cell surface, or complexed to proteoglycan, which would also make detection difficult. ${ }^{26}$ However, we would have expected to detect expression if in the same range as previously reported by Reed et al $(1518 \pm 635 \mathrm{pg} / \mathrm{ml}) .^{7}$ Moreover, we were unable to show increased APRIL mRNA expression in RSV-infected cultured primary AECs despite previous reports of its induction in adult epithelial cells by the TLR3 stimulus, dsRNA. ${ }^{20}$ However, it is possible that a further signal is needed for soluble APRIL expression, or that APRIL is expressed as a protein only at time points later than $48 \mathrm{~h}$, or even that APRIL expression is inhibited by infection with whole virus. In the absence of APRIL, reduced $\mathrm{B}$ cell maturation and memory cell responses might be expected to occur, and enhanced allergic airway responses have been reported. ${ }^{27}$ Interestingly, both incomplete long-term memory responses and persistent respiratory symptoms are both seen subsequent to human RSV disease.

Much further work, beyond the remit of this primarily clinically based study, is needed to fully define the role of BAFF and similar factors in the human airway immune response. That $\mathrm{B}$ lymphocytes are present and are the main type of lymphocyte located within lung tissue during RSV infection has been reported previously. ${ }^{5} 28$ How AECs, through production of BAFF and other factors, influence B cell activity in vivo, and the production of pathogen-specific antibody, requires further investigation. Promoting the formation of, or activity within, 
inducible bronchial associated lymphoid tissue is one possibility. ${ }^{29}$ Another is in driving non-antigen-specific polyclonal responses by B-1 cells, a subpopulation of B cells involved in T cell independent and innate-like immune responses. In murine models of influenza infection, B-1 cells produce a broad repertoire of polyreactive antibodies thought capable of providing protection against secondary infection with other pathogens that threaten an already injured mucosal tissue. ${ }^{30-32}$

AEC BAFF expression, as a feature of both the upper and lower respiratory tract response to infection, at least in infants and young children, has potentially important implications. It strongly supports the contention that a major part of the airway response to infection includes local production of factors that regulate B cell fate. Moreover, it underlines the importance of AECs in driving, not only inflammatory and immune cell recruitment in response to infection, ${ }^{33}$ but also antibody production. A similar role in producing BAFF has been suggested for dendritic cells within the gut mucosa, where it is believed to support B cell IgA production. ${ }^{34}$ Our findings might also be relevant to prospective vaccine design. BAFF, as an airway adjuvant, has been successfully used to enhance murine airway antibody responses to heat-killed pseudomonas. ${ }^{35}$ Our study would seem to suggest that an inhaled adjuvant capable of enhancing the AEC BAFF response would have the potential to augment airway mucosal immunity.

Acknowledgements The authors would like to acknowledge the assistance of Mrs $\mathrm{H}$ Nelson and Mr J Ryding in preparation of the manuscript and figures, $\mathrm{P}$ Bezerra and $\mathrm{M}$ do Carmo Duarte for assistance with the collection and preparation of NPA samples.

Contributions PSM, AF, BFF, RLS, conceived and designed experiments. AF, BFF, $D H, W A$, performed experiments. AF, PSM, DH, BFF analysed the data. RET, JRS, $\mathrm{PSM}, \mathrm{JBC}, \mathrm{AF}, \mathrm{BFF}$, collected samples, contributed materials or analysis tools. BFF, AMF, PSM and RLS wrote the paper

Funding This work was supported by a Wellcome Trust award to PSM and Alder Hey Children's Imagine Appeal supporting AF and RET, a research development award from the Liverpool cancer research centre to J R Slupsky and B F Flanagan. Brazil's National Council for Scientific and Technological Development (Grant number 479381/2006-2) and IMIP's Fund for Teaching and Research supported aspects of this study.

Competing interests None.

Ethics approval The study was approved by the local ethics committees in Liverpool and Recife.

Provenance and peer review Not commissioned; externally peer reviewed.

\section{REFERENCES}

1 Nair H, Nokes DJ, Gessner BD, et al. Global burden of acute lower respiratory infections due to respiratory syncytial virus in young children: a systematic review and meta-analysis. Lancet 2010;375:1545-55

2 Fonceca $A M$, Flanagan $B F$, Trinick $R$, et al. Primary airway epithelial cultures from children are highly permissive to respiratory syncytial virus infection. Thorax 2012:67:42-8.

3 McNamara PS, Flanagan BF, Selby AM, et al. Pro- and anti-inflammatory responses in respiratory syncytial virus bronchiolitis. Eur Respir J : Offic J Eur Soc Clin Respir Physiol 2004;23:106-12.

4 Wang H, Su Z, Schwarze J. Healthy but not RSV-infected lung epithelial cells profoundly inhibit T cell activation. Thorax 2009;64:283-90.

5 Welliver TP, Garofalo RP, Hosakote Y, et al. Severe human lower respiratory tract illness caused by respiratory syncytial virus and influenza virus is characterized by the absence of pulmonary cytotoxic lymphocyte responses. J Infect Dis 2007;195:1126-36.

6 McNamara PS, Ritson P, Selby A, et al. Bronchoalveolar lavage cellularity in infants with severe respiratory syncytial virus bronchiolitis. Arch Dis Child 2003;88:922-6.

7 Reed JL, Welliver TP, Sims GP, et al. Innate immune signals modulate antiviral and polyreactive antibody responses during severe respiratory syncytial virus infection. J Infect Dis 2009:199:1128-38.
8 Bossen C, Schneider P. BAFF, APRIL and their receptors: structure, function and signaling. Semin Immunol 2006;18:263-75.

9 Elgueta R, de Vries VC, Noelle RJ. The immortality of humoral immunity. Immunol Rev 2010;236:139-50.

10 Lied GA, Berstad A. Functional and clinical aspects of the B-cell-activating factor (BAFF): a narrative review. Scand J Immunol 2011;73:1-7.

11 Mackay F, Figgett WA, Saulep D, et al. B-cell stage and context-dependent requirements for survival signals from BAFF and the B-cell receptor. Immunol Rev 2010;237:205-25.

12 Khan WN. B cell receptor and BAFF receptor signaling regulation of B cell homeostasis. J Immunol 2009;183:3561-7.

13 Murphy BR, Graham BS, Prince GA, et al. Serum and nasal-wash immunoglobulin $G$ and $A$ antibody response of infants and children to respiratory syncytial virus $\mathrm{F}$ and $\mathrm{G}$ glycoproteins following primary infection. J Clin Microbiol 1986;23:1009-14.

14 Walsh EE, Falsey AR. Humoral and mucosal immunity in protection from natural respiratory syncytial virus infection in adults. J Infect Dis 2004;190: 373-8.

15 Weltzin R, Traina-Dorge V, Soike K, et al. Intranasal monoclonal IgA antibody to respiratory syncytial virus protects rhesus monkeys against upper and lower respiratory tract infection. J Infect Dis 1996;174:256-61.

16 Falsey AR, Singh HK, Walsh EE. Serum antibody decay in adults following natural respiratory syncytial virus infection. J Med Virol 2006;78:1493-7.

17 McNamara PS, Kicic A, Sutanto EN, et al. Comparison of techniques for obtaining lower airway epithelial cells from children. Eur Respir J: Offic J Eur Soc Clin Respir Physiol 2008;32:763-8.

18 Bezerra PG, Britto MC, Correia JB, et al. Viral and atypical bacterial detection in acute respiratory infection in children under five years. PloS One 2011;6:e18928.

19 Kicic A, Sutanto EN, Stevens PT, et al. Intrinsic biochemical and functional differences in bronchial epithelial cells of children with asthma. Am J Respir Crit Care Med 2006;174:1110-18.

20 Kato A, Truong-Tran AQ, Scott AL, et al. Airway epithelial cells produce B cell-activating factor of TNF family by an IFN-beta-dependent mechanism. J Immunol 2006:177:7164-72.

21 Zhang L, Collins PL, Lamb RA, et al. Comparison of differing cytopathic effects in human airway epithelium of parainfluenza virus 5 (W3A), parainfluenza virus type 3, and respiratory syncytial virus. Virology 2011;421:67-77.

22 Spann KM, Tran KC, Chi B, et al. Suppression of the induction of alpha, beta, and lambda interferons by the NS1 and NS2 proteins of human respiratory syncytial virus in human epithelial cells and macrophages [corrected]. J Virol 2004;78:4363-9.

23 Spann KM, Tran KC, Collins PL. Effects of nonstructural proteins NS1 and NS2 of human respiratory syncytial virus on interferon regulatory factor 3, NF-kappaB, and proinflammatory cytokines. J Virol 2005;79:5353-62.

24 Munir S, Hillyer $P$, Le Nouen $C$, et al. Respiratory syncytial virus interferon antagonist NS1 protein suppresses and skews the human T lymphocyte response. PLoS Pathog 2011;7:e1001336.

25 Munir S, Le Nouen C, Luongo C, et al. Nonstructural proteins 1 and 2 of respiratory syncytial virus suppress maturation of human dendritic cells. J Virol 2008:82:8780-96.

26 Reijmers RM, Groen RW, Kuil A, et al. Disruption of heparan sulfate proteoglycan conformation perturbs B-cell maturation and APRIL-mediated plasma cell survival. Blood 2011;117:6162-71.

27 Xiao Y, Motomura S, Deyev V, et al. TNF superfamily member 13, APRIL, inhibits allergic lung inflammation. Eur J Immunol 2011;41:164-71.

28 Welliver TP, Reed JL, Welliver RC Sr. Respiratory syncytial virus and influenza virus infections: observations from tissues of fatal infant cases. Pediatr Infect Dis $J$ 2008;27(10 Suppl):S92-6.

29 Foo SY, Phipps S. Regulation of inducible BALT formation and contribution to immunity and pathology. Mucosal Immunol 2010;3:537-44.

30 Waffarn EE, Baumgarth N. Protective B cell responses to flu-no fluke! J Immunol 2011;186:3823-9.

31 Choi YS, Baumgarth N. Dual role for B-1a cells in immunity to influenza virus infection. J Exp Med 2008;205:3053-64.

$32 \mathrm{Ng} \mathrm{LG}, \mathrm{Ng} \mathrm{CH}$, Woehl B, et al. BAFF costimulation of Toll-like receptor-activated B-1 cells. Eur J Immunol 2006;36:1837-46.

33 Salvi S, Holgate ST. Could the airway epithelium play an important role in mucosal immunoglobulin A production? Clin Exp Allergy 1999;29:1597-605.

34 Cerutti A, Chen K, Chorny A. Immunoglobulin responses at the mucosal interface. Annu Rev Immunol 2011:29:273-93.

35 Tertilt C, Joh J, Krause A, et al. Expression of B-cell activating factor enhances protective immunity of a vaccine against Pseudomonas aeruginosa. Infect Immun $2009 ; 77: 3044-55$. 